\title{
On the Dynamical Behaviors of a Cholera Model with Holling Type II Functional Response
}

\author{
Ahmed A. Muhseen* and Xueyong Zhou* ${ }^{* *}$ \\ *Ministry of Education, Rusafa, 1, Baghdad-Iraq. \\ ${ }^{* *}$ College of Mathematics and Information Science, Xinyang Normal University, \\ Xinyang 464000, Henan, P.R. China. \\ E-mail: aamuhseen@gmail.com. \\ E-mail: xueyongzhou@126.com.
}

\begin{abstract}
In this paper a mathematical model that describes the flow of Cholera disease in a population is proposed and studied. It is assumed that the disease divided the population into five classes: susceptible individuals $(S)$, asymptomatic infectious individuals $\left(I_{A}\right)$, symptomatic infectious individuals $\left(I_{S}\right)$, removal individuals $(R)$ and cholera population $(B)$. The existence, uniqueness and boundedness of the solution of the model are discussed. The local and global stability of the model is studied. Finally the global dynamics of the proposed model is studied numerically.
\end{abstract}

Keywords: Cholera model, Basic reproduction number, Holling Type II, Local and Global Stability.

\section{Introduction}

Cholera is an acute intestinal infectious disease caused by bacterium Vibrio Cholera. Recent Cholera outbreaks in Haiti (20102011), Nigeria (2010), Kenya (2010), Vietnam (2009), Zimbabwe (2008-2009), and Iraq (2007), etc. The container is leading to a large number of infections and receiving worldwide attention. Then, despite of many clinical and theoretical studies [1-8] and tremendous administrative efforts and interventions, Cholera remains a significant threat to public health in developing countries.

In the year 2006 alone, about 240,000 Cholera cases were officially notified to the World Health Organization (WHO). A deep understanding of the disease dynamic would provide important guidelines to the effective prevention and control strategies [9, 10]. Mathematical modeling, simulation and analysis offer a promising way to look into the natural of Cholera dynamics, and many efforts have been devoted to this topic.

Below, we briefly review some representative mathematical models proposed by various authors. For example, Capasso and Paveri-Fontana [11], introduced a simple deterministic model in 1979 to study a Cholera epidemic in the Mediterranean. In 2001, Codeco [12], extended the model of Capasso and Paveri-Fontana. He added an equation for the dynamics of the susceptible population. In [13], Pascual et al.

Generalized Codeco model by including a fourth equation for the volume of water in which the formative live following Codeco [12]. In 2009, Richard I. Joh et al. considered the dynamic of infectious disease for which the primary mode of transmission is indirect and mediated by contact with a contaminated reservoir [14]. In [15], Rachal L. Miller et al. formulated a mathematical model to include essential components such as a hyperinfectious, a short-lived bacterial state, a separate class for mild human infections, and waning disease immunity. In this paper we proposed and studied a mathematical model of Cholera disease, in which it is assumed that the disease transmitted by contact by Holling Type II functional response. The local as well as global stability analysis of this model is investigated.

\section{Mathematical Model}

Let $S(t), I_{A}(t), I_{B}(t)$ and $R(t)$ be the number of the susceptible individuals, asymptomatic infectious individuals, symptomatic infectious individuals and removal individuals from infected classes at time $t$ respectively. Let $B(t)$ be the cholera population at time $t$ with grows logistically. The state equations, which cover this model, can be written as follows: 


$$
\left.\begin{array}{l}
\dot{S}=\theta-\frac{\beta_{1} S B}{K_{1}+B}-d S \\
\dot{I}_{A}=\frac{\rho \beta_{1} S B}{K_{1}+B}-\left(\gamma_{A}+d\right) I_{A} \\
\dot{I}_{S}=\frac{(1-\rho) \beta_{1} S B}{K_{1}+B}-\left(\gamma_{S}+d+\mu\right) I_{S} \\
\dot{R}=\gamma_{A} I_{A}+\gamma_{S} I_{S}-d R \\
\dot{B}=r B\left(1-\frac{B}{K_{2}}\right)-\eta B+\zeta_{1} I_{A}+\zeta_{2} I_{S}
\end{array}\right\}
$$

Note that all the parameters of system (1) are assumed to be positive constants and can describe as following: $\theta$ birth rate in susceptible class, assumed that the disease transmitted from class $S$ to classes $I_{A}$ and $I_{S}$ by contact according to Holling types II interaction between $S$ class and $B$ class with infection rate constant $\beta_{1}$ with fraction $\rho$ such that $(0 \leq \rho \leq 1), d$ is the natural death rate in each class while the $\mu, \eta$ are the disease related death from $I_{S}$ and $B$ respectively. $\gamma_{A}, \gamma_{S}$ represents the recovery rate constant. $r$ and $K_{2}$ are respectively, the intrinsic growth rate and carrying capacity of cholera population, finally, $\zeta_{1}$ and $\zeta_{2}$ are the new infected members arriving into the cholera population in unit time from $I_{A}$ and $I_{S}$ classes. Therefore, at any point of time $t$ the total number of population becomes

$N=S(t)+I_{A}(t)+I_{S}(t)+R(t)+B(t)$.

Obviously, due to the biological meaning of the variables $S(t), I_{A}(t), I_{S}(t), R(t)$ and $B(t)$, system (1) has the domain

$$
\begin{aligned}
& \mathfrak{R}_{+}^{5}=\left\{\left(S, I_{A}, I_{S}, R, B \in \mathfrak{R}_{+}^{5}, S \geq 0, I_{A} \geq 0,\right.\right. \\
& \left.I_{S} \geq 0, R \geq 0, B \geq 0\right\}, \quad \text { which is positive }
\end{aligned}
$$
invariant for system (1). Clearly, the interaction functions on the right hand side of system (1) are continuously differentiable. In fact they are Liptschizan function on $\mathfrak{R}_{+}^{5}$. Therefore the solution of system (1) exists and unique. Further, all solutions are uniformly bounded as shown in the following theorem:

\section{Theorem (1):}

All the solutions of system (1), which are initiate in $\mathfrak{R}_{+}^{5}$ if exists, are uniformly bounded.

\section{Proof:}

Let $\left(S(t), I_{A}(t), I_{S}(t), R(t), B(t)\right)$ be any solution of system (1) with non-negative initial condition $\left(S(0), I_{A}(0), I_{S}(0), R(0), B(0)\right)$, since $N(t)=S(t)+I_{A}(t)+I_{S}(t)+R(t)+B(t)$, then:

$$
\dot{N}=\dot{S}+\dot{I}_{A}+\dot{I}_{S}+\dot{R}+\dot{B}
$$

this gives

$$
\begin{aligned}
\dot{N}= & \theta-d S-\left(d-\zeta_{1}\right) I_{A}-\left(d-\zeta_{2}\right) I_{S}-d R \\
& -(\eta-r) B-\mu I_{S}-\frac{r B^{2}}{K_{2}} \\
\dot{N} \leq & \theta-m N,
\end{aligned}
$$

where $m=\min .\left\{d,\left(d-\zeta_{1}\right),\left(d-\zeta_{2}\right),(\eta-r)\right\}$

$$
\dot{N}+m N \leq \theta
$$

Now, by using Gronwall lemma [16], it obtains that:

$$
N(t) \leq \frac{\theta}{\mu}\left(1-e^{-\mu t}\right)+N(0) e^{-\mu t}
$$

Therefore, $N(t) \leq \frac{\theta}{\mu}$, as $t \rightarrow \infty$, hence all the solutions of system (1) that initiate in $\mathfrak{R}_{+}^{5}$ are confined in the region:

$$
\tau=\left\{\left(S, I_{A}, I_{S}, R, B\right) \in \mathfrak{R}_{+}^{5}: N \leq \frac{\theta}{\mu}\right\}
$$

Which is complete the proof.

\section{The Basic Reproduction Number}

For all infectious disease, the basic reproduction number, sometimes called basic reproductive ratio, is one of the most useful threshold parameters that characterizes mathematical problems concerning infectious disease. This metric is useful because it helps us to determine whether an infectious disease will spread through a population, we will calculate the basic reproduction number.

It easy to see that this system always has a disease free equilibrium point (the absence of infection, that is, $\left.I_{A}=I_{S}=B=0\right)$,

$E_{0}=\left(S_{0}, 0,0,0\right)$, where $S_{0}=\frac{\theta}{d}$. Let 


$$
\begin{gathered}
X=\left(I_{A}, I_{S}, B, S\right)^{T} . \text { Then, we get: } \\
\dot{X}=f(x)-v(x)
\end{gathered}
$$

where

$$
\begin{aligned}
& f(x)=\left(\begin{array}{c}
\frac{\rho \beta_{1} S B}{K_{1}+B} \\
\frac{(1-\rho) \beta_{1} S B}{K_{1}+B} \\
r B\left(1-\frac{B}{\left.K_{2}\right)}\right. \\
0
\end{array}\right) \\
& v(x)=\left(\begin{array}{c} 
\\
\left(\gamma_{A}+d\right) I_{A} \\
\left(\gamma_{S}+d+\mu\right) I_{S} \\
\eta B-\zeta_{1} I_{A}-\zeta_{2} I_{S} \\
\frac{\beta_{1} S B}{K_{1}+B}+d S-\theta
\end{array}\right)
\end{aligned}
$$

We can obtain:

$$
\begin{aligned}
& F=\left(\begin{array}{ccc}
0 & 0 & \frac{\rho \beta_{1} S_{0}}{K_{1}} \\
0 & 0 & \frac{(1-\rho) \beta_{1} S_{0}}{K_{1}} \\
0 & 0 & r
\end{array}\right) \\
& V=\left(\begin{array}{ccc}
\gamma_{A}+d & 0 & 0 \\
0 & \gamma_{S}+d+\mu & 0 \\
-\zeta_{1} & -\zeta_{2} & \eta
\end{array}\right),
\end{aligned}
$$

giving

$$
V^{-1}=\left(\begin{array}{ccc}
\frac{1}{\gamma_{A}+d} & 0 & 0 \\
0 & \frac{1}{\gamma_{S}+d+\mu} & 0 \\
\frac{\zeta_{1}}{\eta\left(\gamma_{A}+d\right)} & \frac{\zeta_{2}}{\eta\left(\gamma_{S}+d+\mu\right)} & \frac{1}{\eta}
\end{array}\right)
$$

$F V^{-1}$ is the next-generation matrix for model (3). It then follows that the spectral radius of matrix $F V^{-1}$ is

$$
\begin{aligned}
\rho\left(F V^{-1}\right)= & \frac{r}{\eta}\left(\frac{\rho \zeta_{1} \beta_{1} S_{0}}{\eta K_{1}\left(\gamma_{A}+d\right)}\right) . \\
& \left(\frac{(1-\rho) \zeta_{2} \beta_{1} S_{0}}{\eta K_{1}\left(\gamma_{S}+d+\mu\right)}\right)
\end{aligned}
$$

According to theorem 2 in [17], the basic reproduction number of model (3) is:

$\mathfrak{R}_{\circ}=\frac{r \theta \beta_{1}}{\eta^{3} d K_{1}}\left(\frac{\rho \zeta_{1}}{\gamma_{A}+d}\right)\left(\frac{(1-\rho) \zeta_{2}}{\gamma_{S}+d+\mu}\right)$

\section{Existence of Equilibrium Points of System (1)}

In this section, we shall discuss the existence of all possible equilibrium points of system (1). Now since recovery class $R$ is related with infected classes $I_{A}$ and $I_{S}$ only, hence knowing the values of $I_{A}$ and $I_{S}$ leads directly to determine the value of $R$ from solving the fifth equation in system (1). In fact, if the $I_{i}=0, i=A, S$, then $R$ approaches to zero asymptotically. However, if $I_{A}=I_{c}$ and $I_{S}=I_{k}$ where $I_{c}$ and $I_{k}$ are positive constant, then $R$ approaches to:

$$
R=\frac{\gamma_{A} I_{c}+\gamma_{S} I_{k}}{d}
$$

Consequently, system (1) can be written as below and then equation (3) can be used to give the value of $R$.

$$
\left.\begin{array}{l}
\dot{S}=\theta-\frac{\beta_{1} S B}{K_{1}+B}-d S \\
\dot{I}_{A}=\frac{\rho \beta_{1} S B}{K_{1}+B}-\left(\gamma_{A}+d\right) I_{A} \\
\dot{I}_{S}=\frac{(1-\rho) \beta_{1} S B}{K_{1}+B}-\left(\gamma_{S}+d+\mu\right) I_{S} \\
\dot{B}=r B\left(1-\frac{B}{K_{2}}\right)-\eta B+\zeta_{1} I_{A}+\zeta_{2} I_{S}
\end{array}\right\}
$$

Now, system (4) has at most two biologically feasible points, namely $E_{i}=\left(S_{i}, I_{A i}, I_{S i}, B_{i}\right)$, $i=0,1$. The existence conditions for each of these equilibrium points are discussed in following:

1) If $I_{A}=0, I_{S}=0, B=0$ and $\mathfrak{R}_{\circ}<1$, then system (4) has an equilibrium point called disease free equilibrium point and denoted by $E_{0}=\left(S_{0}, 0,0,0\right)$ where:

$S_{0}=\frac{\theta}{d}$

2) If $I_{A} \neq 0, I_{S} \neq 0, B \neq 0$, and $\mathfrak{R}_{\circ}>1$, then system (4) has an equilibrium point called endemic equilibrium point and denoted by 
$E_{1}\left(S_{1}, I_{A 1}, I_{S 1}, B_{1}\right) \quad$ where $S_{1}, I_{A 1}, I_{S 1}$ and $B_{1}$ represent the positive solution of following set of equations:

$$
\left.\begin{array}{l}
\theta-\frac{\beta_{1} S B}{K_{1}+B}-d S=0 \\
\frac{\rho \beta_{1} S B}{K_{1}+B}-\left(\gamma_{A}+d\right) I_{A}=0 \\
\frac{(1-\rho) \beta_{1} S B}{K_{1}+B}-\left(\gamma_{S}+d+\mu\right) I_{S}=0 \\
B\left[r-\frac{B r}{K_{2}}-\eta\right]+\zeta_{1} I_{A}+\zeta_{2} I_{S}=0
\end{array}\right\}
$$

Obviously, from $1^{\text {st }}, 2^{\text {nd }}$ and $3^{\text {rd }}$ equations of (6) we get:

$$
\begin{aligned}
& S_{1}=\frac{\theta\left(K_{1}+B_{1}\right)}{G} \ldots \ldots \ldots \ldots \ldots \ldots \ldots \ldots \ldots \ldots \\
& I_{A 1}=\frac{\rho \theta \beta_{1}\left(K_{1}+B_{1}\right) B_{1}}{\left(K_{1}+B_{1}\right)\left(\gamma_{A}+d\right) G} \ldots \ldots . \\
& I_{S 1}=\frac{(1-\rho) \beta_{1} \theta\left(K_{1}+B_{1}\right) B_{1}}{\left(K_{1}+B_{1}\right)\left(\gamma_{S}+d+\mu\right) G}
\end{aligned}
$$

Where $G=\beta_{1} B_{1}+d\left(K_{1}+B_{1}\right)$

Substituting $I_{A 1}$ and $I_{S 1}$ in the $4^{\text {th }}$ equation of (6) we get:

$$
\Omega_{1} B_{1}^{4}+\Omega_{2} B_{1}^{3}+\Omega_{3} B_{1}^{2}+\Omega_{4} B_{1}=0
$$

Here:

$$
\begin{gathered}
\Omega_{1}=-r\left(\beta_{1}+d\right)\left(\gamma_{A}+d\right)\left(\gamma_{S}+d+\mu\right)<0 \\
\Omega_{2}=\left(\gamma_{A}+d\right)\left(\gamma_{S}+d+\mu\right) \cdot \\
{\left[r K_{2}\left(\beta_{1}+d\right)-\left(r K_{1}\left(\beta_{1}+2 d\right)+\eta K_{2}\left(\beta_{1}+d\right)\right)\right]} \\
\Omega_{3}=\theta \beta_{1} K_{2}\left[\rho \zeta_{1}\left(\gamma_{S}+d+\mu\right)\right. \\
\left.+\zeta_{2}(1-\rho)\left(\gamma_{A}+d\right)\right]+r K_{1} K_{2}\left(\beta_{1}+2 d\right) . \\
\quad\left(\gamma_{A}+d\right)\left(\gamma_{S}+d+\mu\right)-\left[\eta K_{1} K_{2} .\right. \\
\left(\beta_{1}+2 d\right)\left(\gamma_{A}+d\right)\left(\gamma_{S}+d+\mu\right) \\
\left.\quad+r K_{1}^{2} d\left(\gamma_{A}+\gamma_{S}+2 d+\mu\right)\right] \\
\Omega_{4}=\beta_{1} \theta K_{1} K_{2}\left[\rho \zeta_{1}\left(\gamma_{S}+d+\mu\right)+\zeta_{2}(1-\rho)\right. \\
\left.\left(\gamma_{A}+d\right)\right]+d K_{1}^{2} K_{2}(r-\eta) . \\
{\left[\gamma_{A}+\gamma_{S}+2 d+\mu\right]}
\end{gathered}
$$

Clearly, equation (7d) by Descartes rule [18] has a unique positive root given by $B_{1}$ and then the equilibrium point $\left(E_{1}\right)$ exists uniquely in Int. $\mathfrak{R}_{+}^{4}$ if and only if the $\Omega_{4}>0$ (positive) then we have the following three Cases:

\section{Case (1):}

If the following conditions are hold:

$\left.\begin{array}{l}\Omega_{2}>0 \\ \Omega_{3}>0\end{array}\right\}$

Case (2): If the following conditions are hold: $\left.\Omega_{2}<0\right\}$

$\left.\Omega_{3}<0\right\}$

Case (3): If the following conditions are hold: $\left.\Omega_{2}<0\right\}$ $\left.\Omega_{3}>0\right\}$

\section{Local Stability Analysis of System (4)}

In this section, the local stability analysis of the each equilibrium points $E_{i}, i=0,1$ of system (4) studied as shown in the following theorems.

\section{Theorem (2):}

The asymptomatic and symptomatic infectious free equilibrium point $E_{0}=\left(S_{0}, 0,0,0\right)$ of System (4) is locally asymptotically stable when $\mathfrak{R}_{\circ}<1$ and then the following conditions are satisfied, but $E_{0}$ unstable when $\mathfrak{R}_{\circ}>1$ :

$$
\begin{aligned}
& d>\max .\left\{\zeta_{1}-\gamma_{A}, \zeta_{2}-\left(\gamma_{S}+\mu\right)\right\} \\
& \eta K_{1}>2 \beta_{1} K_{1} S_{0}+r K_{1} \ldots \ldots \ldots \ldots \ldots \ldots
\end{aligned}
$$

\section{Proof:}

The Jacobian matrix of system (4) at $\left(E_{0}\right)$ that denoted by $J\left(E_{0}\right)$ and can be written:

$$
J\left(E_{0}\right)=\left[a_{i j}\right]_{4 \times 4},
$$

where:

$$
\begin{aligned}
& a_{11}=-d ; \quad a_{14}=-\frac{\beta_{1} S_{0}}{K_{1}} ; \quad a_{22}=-\left(\gamma_{A}+d\right) ; \\
& a_{24}=\frac{\rho \beta_{1} S_{0}}{K_{1}} ; \quad a_{33}=-\left(\gamma_{S}+d+\mu\right) ; \\
& a_{34}=\frac{(1-\rho) \beta_{1} S_{0}}{K_{1}} ; \quad a_{42}=\zeta_{1} ; \quad a_{43}=\zeta_{2} ;
\end{aligned}
$$

$a_{44}=r-\eta$ and zero otherwise.

Now, according to Gershgorin theorem [19] if the following condition hold: 


$$
\left|a_{i i}\right|>\sum_{\substack{i=1 \\ i \neq j}}^{4}\left|a_{i j}\right|
$$

Then all eigenvalues of $J\left(E_{0}\right)$ exists in the region:

$$
\Lambda=\bigcup\left\{U^{*} \in C:\left|U^{*}-a_{i i}\right|<\sum_{\substack{i=j \\ i \neq j}}^{4}\left|a_{i j}\right|\right\}
$$

Therefore, according to the given conditions (9a), (9b) all the eigenvalues of $J\left(E_{0}\right)$ exists in the left half plane and hence, $\left(E_{0}\right)$ is locally asymptotically stable.

\section{Theorem (3):}

The endemic equilibrium point $E_{1}=\left(S_{1}, I_{A 1}, I_{S 1}, B_{1}\right)$ of System (4) is locally asymptotic stable when $\mathfrak{R}_{\circ}>1$ and then the following conditions are satisfied:

$$
\begin{gathered}
{\left[\frac{\beta_{1} K_{1} S_{1}}{\left(K_{1}+B_{1}\right)^{2}}\right]^{2}<\frac{4}{9}\left[\frac{\beta_{1} B_{1}}{K_{1}+B_{1}}+d\right]} \\
\cdot\left[\frac{2 r B_{1}}{\left(K_{1}+B_{1}\right)^{2}}+\eta-r\right] \\
{\left[\frac{\rho \beta_{1} B_{1}}{K_{1}+B_{1}}\right]^{2}<\frac{2}{3}\left[\frac{\beta_{1} B_{1}}{K_{1}+B_{1}}+d\right] .}
\end{gathered}
$$

$$
\left[\gamma_{A}+d\right]
$$$$
\left[\frac{(1-\rho) \beta_{1} B_{1}}{K_{1}+B_{1}}\right]^{2}<\frac{2}{3}\left[\frac{\beta_{1} B_{1}}{K_{1}+B_{1}}+d\right] \text {. }
$$$$
\left[\gamma_{S}+d+\mu\right]
$$

$$
\left[\frac{\rho \beta_{1} K_{1} S_{1}}{\left(K_{1}+B_{1}\right)^{2}}+\zeta_{1}\right]^{2}<\frac{2}{3}\left[\gamma_{A}+d\right]
$$

$$
\left[\frac{2 r B_{1}}{K_{2}}+\eta-r\right]
$$

$$
\begin{array}{r}
{\left[\frac{(1-\rho) \beta_{1} K_{1} S_{1}}{\left.\left(K_{1}+B_{1}\right)^{2}+\zeta_{2}\right]^{2}}<\frac{2}{3}\left[\gamma_{S}+d+\mu\right]\right.} \\
\cdot\left[\frac{2 r B_{1}}{K_{2}}+\eta-r\right]
\end{array}
$$

$r<\frac{2 r B_{1}}{K_{2}}+\eta$

\section{Proof:}

The Jacobian matrix of System (4) at $E_{1}=\left(S_{1}, I_{A 1}, I_{S 1}, B_{1}\right)$ written by:

$J\left(E_{1}\right)=\left[z_{i j}\right]_{4 \times 4}$, where:

$z_{11}=-\left(\frac{\beta_{1} B_{1}}{K_{1}+B_{1}}+d\right) ; \quad z_{14}=-\frac{\beta_{1} K_{1} S_{1}}{\left(K_{1}+B_{1}\right)^{2}} ;$

$z_{21}=\frac{\rho \beta_{1} B_{1}}{K_{1}+B_{1}} ; z_{22}=-\left(\gamma_{A}+d\right)$

$z_{24}=\frac{\rho \beta_{1} K_{1} S_{1}}{\left(K_{1}+B_{1}\right)^{2}} ; \quad z_{31}=\frac{(1-\rho) \beta_{1} B_{1}}{K_{1}+B_{1}} ;$

$z_{33}=-\left(\gamma_{S}+d+\mu\right) ; z_{34}=\frac{(1-\rho) \beta_{1} K_{1} S_{1}}{\left(K_{1}+B_{1}\right)^{2}}$

$z_{42}=\zeta_{1} ; z_{43}=\zeta_{2} ; \quad z_{44}=r-\left(\frac{2 r B_{1}}{K_{2}}+\eta\right)$ and zero otherwise.

It is easy to verify that the linearization system of system (4) can be written as:

$\dot{N}=\dot{X}=J\left(E_{1}\right) \cdot X$

Here, $N=\left(S, I_{A}, I_{S}, B\right)^{T}$ and

$X=\left(x_{1}, x_{2}, x_{3}, x_{4}\right)^{T}$, where:

$x_{1}=S-S_{1} ; x_{2}=I_{A}-I_{A 1} ; x_{3}=I_{S}-I_{S 1}$;

$x_{4}=B-B_{1}$.

Now, consider the following positive definite function:

$V=\frac{x_{1}^{2}}{2}+\frac{x_{2}^{2}}{2}+\frac{x_{3}^{2}}{2}+\frac{x_{4}^{2}}{2}$

It is clearly that $V: R_{+}^{4} \rightarrow R$ and a continuously differentiable function so that $V\left(S_{1}, I_{A 1}, I_{S 1}, B_{1}\right)=0$ and $V\left(S, I_{A}, I_{S}, B\right)>0$ otherwise. So by differentiating $V$ with respect to time $t$, gives:

$\dot{V}=x_{1} \cdot \dot{X}_{1}+x_{2} \cdot \dot{X}_{2}+x_{3} \cdot \dot{X}_{3}+x_{4} \cdot \dot{X}_{4}$

Substituting the values of $\dot{X}_{1}, \dot{X}_{2}, \dot{X}_{3}$ and $\dot{X}_{4}$ in the above equation, and after doing some algebraic manipulation; we get that: 


$$
\begin{aligned}
\dot{V}= & -\frac{1}{3}\left[\frac{\beta_{1} B_{1}}{K_{1}+B_{1}}+d\right] x_{1}^{2}-\left[\frac{\beta_{1} K_{1} S_{1}}{\left(K_{1}+B_{1}\right)^{2}} x_{1} x_{4}\right] \\
- & \frac{1}{3}\left[\frac{2 r B_{1}}{K_{2}}+\eta-r\right] x_{4}^{2}-\frac{1}{3}\left[\frac{\beta_{1} B_{1}}{K_{1}+B_{1}}+d\right] x_{1}^{2} \\
& +\left[\frac{\rho \beta_{1} B_{1}}{K_{1}+B_{1}}\right] x_{1} x_{2}-\frac{1}{2}\left[\gamma_{A}+d\right] x_{2}^{2} \\
& -\frac{1}{3}\left[\frac{\beta_{1} B_{1}}{K_{1}+B_{1}}+d\right] x_{1}^{2}+\left[\frac{(1-\rho) \beta_{1} B_{1}}{K_{1}+B_{1}}\right] x_{1} x_{3} \\
& -\frac{1}{2}\left[\gamma_{S}+d+\mu\right] x_{3}^{2}-\frac{1}{2}\left[\gamma_{A}+d\right] x_{2}^{2} \\
& +\left[\frac{\rho \beta_{1} K_{1} S_{1}}{\left(K_{1}+B_{1}\right)^{2}}+\zeta_{1}\right] x_{2} x_{4}-\frac{1}{3}\left[\frac{2 r B_{1}}{K_{2}}+\eta-r\right] x_{4}^{2} \\
& -\frac{1}{2}\left[\gamma_{S}+d+\mu\right] x_{3}^{2}+\left[\frac{(1-\rho) \beta_{1} K_{1} S_{1}}{\left(K_{1}+B_{1}\right)^{2}}+\zeta_{2}\right] x_{3} x_{4} \\
& -\frac{1}{3}\left[\frac{2 r B_{1}}{K_{2}}+\eta-r\right] x_{4}^{2}
\end{aligned}
$$

Now it is easy to verify that the above set of conditions (10a)-(10e) guarantees the quadratic terms given below:

$$
\begin{aligned}
\dot{V} \leq & -\left[\sqrt{\frac{1}{3}\left(\frac{\beta_{1} B_{1}}{K_{1}+B_{1}}+d\right)} x_{1}+\sqrt{\frac{1}{3}\left(\frac{2 r B_{1}}{K_{2}}+\eta-r\right)} x_{4}\right]^{2} \\
& -\left[\sqrt{\frac{1}{3}\left(\frac{\beta_{1} B_{1}}{K_{1}+B_{1}}+d\right)} x_{1}+\sqrt{\frac{1}{2}\left(\gamma_{A}+d\right) x_{2}}\right]^{2} \\
& -\left[\sqrt{\frac{1}{3}\left(\frac{\beta_{1} B_{1}}{K_{1}+B_{1}}+d\right)} x_{1}+\sqrt{\frac{1}{2}\left(\gamma_{S}+d+\mu\right) x_{3}}\right]^{2} \\
& -\left[\sqrt{\frac{1}{2}\left(\gamma_{A}+d\right) x_{2}}+\sqrt{\frac{1}{3}\left(\frac{2 r B_{1}}{K_{2}}+\eta-r\right) x_{4}}\right]^{2} \\
& -\left[\sqrt{\frac{1}{2}\left(\gamma_{S}+d+\mu\right) x_{3}}+\sqrt{\frac{1}{3}\left(\frac{2 r B_{1}}{K_{2}}+\eta-r\right) x_{4}}\right]^{2}
\end{aligned}
$$

So, $\dot{V}$ is a negative definite, and hence $\dot{V}$ is a Lyapunov function. Thus, $\left(E_{1}\right)$ is a local asymptotically stable and the proof is complete.

\section{Global Stability Analysis of System (4)}

In this section, the global stability analysis of the all equilibrium points $E_{i}, i=0,1$ of system (4) studied as shown in the following theorems.

\section{Theorem (4):}

Assume that, the disease free equilibrium point $E_{0}$ of System (4) is locally asymptotically stable. Then the basin of attraction of $\left(E_{0}\right), \quad$ say $Q\left(E_{0}\right) \subset R_{+}^{4}$, it is globally asymptotically stable if satisfy the following condition:

$$
\begin{aligned}
& d>\max .\left\{\zeta_{1}+\gamma_{A}, \zeta_{2}+\gamma_{S}+\mu\right\} \\
& \frac{\eta K_{2}+r B}{K_{2}}>\frac{r\left(K_{1}+B\right)+B_{1} S}{K_{1}+B} \ldots
\end{aligned}
$$

Proof: Consider the following positive definite function:

$V_{1}=\left(S-S_{0}-S_{0} \ln \frac{S}{S_{0}}\right)+I_{A}+I_{S}+B$

Clearly, $\quad V_{1}: R_{+}^{4} \rightarrow R \quad$ is a continuously differentiable function such that $V_{1}\left(S_{0}, 0,0,0\right)=0$, and

$V_{1}\left(S, I_{A}, I_{S}, B\right)>0, \forall\left(S, I_{A}, I_{S} \cdot B\right) \neq\left(S_{0}, 0,0,0\right)$. Further we have:

$$
\dot{V}_{1}=\left(\frac{S-S_{0}}{S}\right) \dot{S}+\dot{I}_{A}+\dot{I}_{S}+\dot{B}
$$

By simplifying this equation we get:

$$
\begin{aligned}
\dot{V}_{1}=- & \frac{d}{S}\left(S-S_{0}\right)^{2}-\frac{\beta_{1} B}{K_{1}+B}\left(S-S_{0}\right) \\
& +\left[\zeta_{1}-\left(\gamma_{A}+d\right)\right] I_{A}+\left[\zeta_{2}-\left(\gamma_{S}+d+\mu\right)\right] I_{S} \\
& +B\left[\left(r+\frac{\beta_{1} S}{K_{1}+B}\right)-\left(\eta+\frac{r B}{K_{2}}\right)\right]
\end{aligned}
$$

Obviously, $\dot{V}_{1}<0$, for every initial points and then $V_{1}$ is a Lyapunov function provided that conditions (11a)-(11b) hold. Thus $E_{0}$ is globally asymptotically stable in the interior of $Q\left(E_{0}\right)$, which means that $Q\left(E_{0}\right)$ is the basin of attraction and that complete the proof.

\section{Theorem (5):}

Let the endemic equilibrium point $E_{1}$ of System (4) is locally asymptotically stable. Then it is globally asymptotically stable provided that:

$r K_{2}<r\left(B+B_{1}\right)+\eta K_{2}$ 
$\left[\frac{\rho \beta_{1} B_{1}}{K_{1}+B_{1}}\right]^{2}<\frac{2}{3}\left[\frac{G}{K_{1}+B_{1}}\right] \cdot\left[\gamma_{A}+d\right]$

$\left[\frac{(1-\rho) \beta_{1} B_{1}}{K_{1}+B_{1}}\right]^{2}<\frac{2}{3}\left[\frac{G}{K_{1}+B_{1}}\right]$.

$$
\begin{array}{r}
{\left[\frac{\beta_{1} K_{1} S_{1}}{\left(K_{1}+B\right)\left(K_{1}+B_{1}\right)}\right]^{2}<\frac{4}{9}\left[\frac{G}{K_{1}+B_{1}}\right] .} \\
{\left[\frac{G^{\prime}}{K_{2}}\right]}
\end{array}
$$$$
\left[\frac{G^{\prime \prime}}{\left(K_{1}+B\right)\left(K_{1}+B_{1}\right)}\right]^{2}<\frac{2}{3}\left[\gamma_{A}+d\right]
$$$$
\cdot\left[\frac{G^{\prime}}{K_{2}}\right]
$$$$
\left[\frac{G^{\prime \prime \prime}}{\left(K_{1}+B\right)\left(K_{1}+B_{1}\right)}\right]^{2}<\frac{2}{3}\left[\gamma_{S}+d+\mu\right] \text {. }
$$$$
\left[\frac{G^{\prime}}{K_{2}}\right]
$$

\section{Where:}

$$
\begin{aligned}
& G=\beta_{1} B_{1}+d\left(K_{1}+B_{1}\right) \\
& G^{\prime}=r\left(B+B_{1}\right)+(\eta-r) K_{2} \\
& G^{\prime \prime}=\rho \beta_{1} K_{1} S_{1}+\zeta_{1}\left(K_{1}+B\right)\left(K_{1}+B_{1}\right) \\
& G^{\prime \prime \prime}=(1-\rho) \beta_{1} K_{1} S_{1}+\zeta_{2}\left(K_{1}+B\right)\left(K_{1}+B_{1}\right)
\end{aligned}
$$

\section{Proof:}

Consider the following positive definite function:

$$
\begin{array}{r}
V_{2}=\frac{\left(S-S_{1}\right)^{2}}{2}+\frac{\left(I_{A}-I_{A 1}\right)^{2}}{2}+\frac{\left(I_{S}-I_{S 1}\right)^{2}}{2} \\
+\frac{\left(B-B_{1}\right)^{2}}{2}
\end{array}
$$

Clearly, $\quad V_{2}: R_{+}^{4} \rightarrow R \quad$ is a continuously differentiable function such that $V_{2}\left(S_{1}, I_{A 1}, I_{S 1}, B_{1}\right)=0 \quad$ and $V_{2}\left(S, I_{A}, I_{S}, B\right)>0, \forall\left(S, I_{A}, I_{S}, B\right) \neq\left(S_{1}, I_{A 1}, I_{S 1}, B_{1}\right)$ Further, we have:

$$
\begin{aligned}
\dot{V}_{2}=\left(S-S_{1}\right) \dot{S}+\left(I_{A}-I_{A 1}\right) \dot{I}_{A}+\left(I_{S}-I_{S 1}\right) \dot{I}_{S} \\
+\left(B-B_{1}\right) \dot{B}
\end{aligned}
$$

By simplifying this equation we get:

$$
\begin{aligned}
\dot{V}_{1}= & -\frac{p_{11}}{3}\left(S-S_{1}\right)^{2}+p_{12}\left(S-S_{1}\right)\left(I_{A}-I_{A 1}\right) \\
& -\frac{p_{22}}{2}\left(I_{A}-I_{A 1}\right)^{2}-\frac{p_{11}}{3}\left(S-S_{1}\right)^{2} \\
& +p_{13}\left(S-S_{1}\right)\left(I_{S}-I_{S 1}\right)-\frac{p_{33}}{2}\left(I_{S}-I_{S 1}\right)^{2} \\
& -\frac{p_{11}}{3}\left(S-S_{1}\right)^{2}+p_{14}\left(S-S_{1}\right)\left(B-B_{1}\right) \\
& -\frac{p_{44}}{3}\left(B-B_{1}\right)^{2}-\frac{p_{22}}{2}\left(I_{A}-I_{A 1}\right)^{2} \\
& +p_{24}\left(I_{A}-I_{A 1}\right)\left(B-B_{1}\right)-\frac{p_{44}}{3}\left(B-B_{1}\right)^{2} \\
& -\frac{p_{33}}{2}\left(I_{S}-I_{S 1}\right)^{2}+p_{34}\left(I_{S}-I_{S 1}\right)\left(B-B_{1}\right) \\
& -\frac{p_{44}}{3}\left(B-B_{1}\right)^{2}
\end{aligned}
$$

With:

$$
\begin{aligned}
& p_{11}=\frac{G}{K_{1}+B_{1}} ; p_{12}=\frac{\rho \beta_{1} B_{1}}{K_{1}+B_{1}} ; p_{22}=\gamma_{A}+d ; \\
& p_{13}=\frac{(1-\rho) \beta_{1} B_{1}}{K_{1}+B_{1}} ; p_{33}=\gamma_{S}+d+\mu ; \\
& p_{14}=\frac{\beta_{1} S_{1} K_{1}}{\left(K_{1}+B\right)\left(K_{1}+B_{1}\right)} ; p_{44}=\frac{G^{\prime}}{K_{2}} ; \\
& p_{24}=\frac{G^{\prime \prime}}{\left(K_{1}+B\right)\left(K_{1}+B_{1}\right)} ; p_{34}=\frac{G^{\prime \prime \prime}}{\left(K_{1}+B\right)\left(K_{1}+B_{1}\right)}
\end{aligned}
$$

Therefore, according to the conditions (12a)(12f) we obtain that:

$$
\begin{aligned}
\dot{V}_{2} \leq- & {\left[\sqrt{\frac{p_{11}}{3}}\left(S-S_{1}\right)-\sqrt{\frac{p_{22}}{2}}\left(I_{A}-I_{A 1}\right)\right]^{2} } \\
-[ & \left.\sqrt{\frac{p_{11}}{3}}\left(S-S_{1}\right)+\sqrt{\frac{p_{33}}{2}}\left(I_{S}-I_{S 1}\right)\right]^{2} \\
& -\left[\sqrt{\frac{p_{11}}{3}}\left(S-S_{1}\right)-\sqrt{\frac{p_{44}}{3}}\left(B-B_{1}\right)\right]^{2} \\
- & {\left[\sqrt{\frac{p_{22}}{2}}\left(I_{A}-I_{A 1}\right)+\sqrt{\frac{p_{44}}{3}}\left(B-B_{1}\right)\right]^{2} } \\
& -\left[\sqrt{\frac{p_{33}}{2}}\left(I_{S}-I_{S 1}\right)+\sqrt{\frac{p_{44}}{3}}\left(B-B_{1}\right)\right]^{2}
\end{aligned}
$$

Clearly, $\dot{V}_{2}<0$, and then $V_{2}$ is a Lyapunov function provided that the given conditions(12a)-(12f) hold. Therefore, $\left(E_{1}\right)$ is globally asymptotically stable. 


\section{Numerical Simulation of System (1)}

In this section, system (1) is solved numerically for different sets of hypothesis data and different sets of initial conditions, and then the time series for the trajectories of system (1) are confirm our obtained analytical results. By using $(0.5,0.7,0.3,0.6,0.9)$ and $(50,40,25,30,0.1)$ as initial points and the numerical simulations are carried out in the following cases:

\section{Case I:}

For the disease free equilibrium point $E_{0}$, we choose the following data:

$$
\begin{aligned}
& \theta=50 ; \beta_{1}=0.0000001 ; K_{1}=0.5 \\
& ; d=0.3 ; \rho=0 ; \gamma_{A}=0.4 ; \gamma_{S}=0.1 \\
& ; \mu=0.01 ; r=0.1 ; K_{2}=0.001 ; \\
& \eta=0.3 ; \zeta_{1}=0.4 ; \zeta_{2}=0.1 ; \Re_{\circ}=0<1
\end{aligned}
$$

Therefore, the disease free equilibrium point $E_{0}$ of system (1) is globally asymptotically stable and is identically to $(167,0,0,0,0)$ for any time. See Fig.(1).

\section{Case II:}

For the endemic equilibrium point $E_{1}$, we choose the following data:

$$
\begin{aligned}
& \theta=50 ; \beta_{1}=0.01 ; K_{1}=0.5 \\
& ; d=0.1 ; \rho=0.1 ; \gamma_{A}=0.2 ; \gamma_{S}=0.001 \\
& ; \mu=0.01 ; r=6 ; K_{2}=50 ; \\
& \eta=7 ; \zeta_{1}=4 ; \zeta_{2}=8 ; \\
& \mathfrak{R}_{\circ}=15.128831>1
\end{aligned}
$$

Therefore, the endemic equilibrium point $E_{1}$ of system (1) is globally asymptotically stable and is identically to $(255,148,36,297,23)$ for any time. See Fig.(2).

\section{Case III:}

We fixed all parameters in equation (14) but we change infection rate value $\beta_{1}=0.01,0.05,0.1,0.2,0.5$ respectively, we get the trajectories of system (1) still approaches to endemic equilibrium point but the number of asymptomatic infectious individuals decrease while the number of the symptomatic infectious and cholera population increases. See Fig.(3a-3c).

\section{Case IV:}

We choose fraction rate $\rho=0,0.2,0.5,0.8,1$ respectively, keeping other parameters fixed as given in equation (14), we get the trajectories of system (1) still approaches to endemic equilibrium point but the number of symptomatic infectious individuals decrease while the number of the asymptomatic infectious and cholera population increases. See Fig.(4a-4c).

\section{Case V:}

Now we choose intrinsic growth rate $r=1,6,15,40$ respectively, keeping other parameters fixed as given in equation (14), we get the trajectories of system (1) still approaches to endemic equilibrium point but the number of asymptomatic infectious individuals and the number of the symptomatic infectious are smoothly decreases while the cholera population is decreases too. See Fig.(5a-5c), and we get inverse above results from increases of carrying capacity rate $\left(K_{2}\right)$.
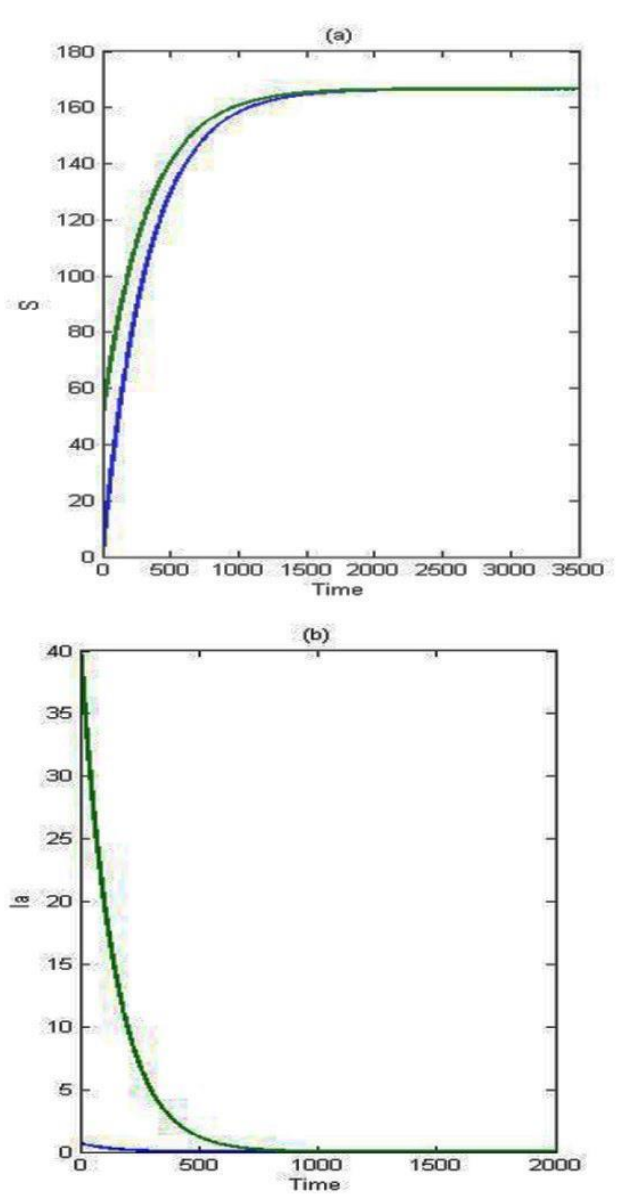

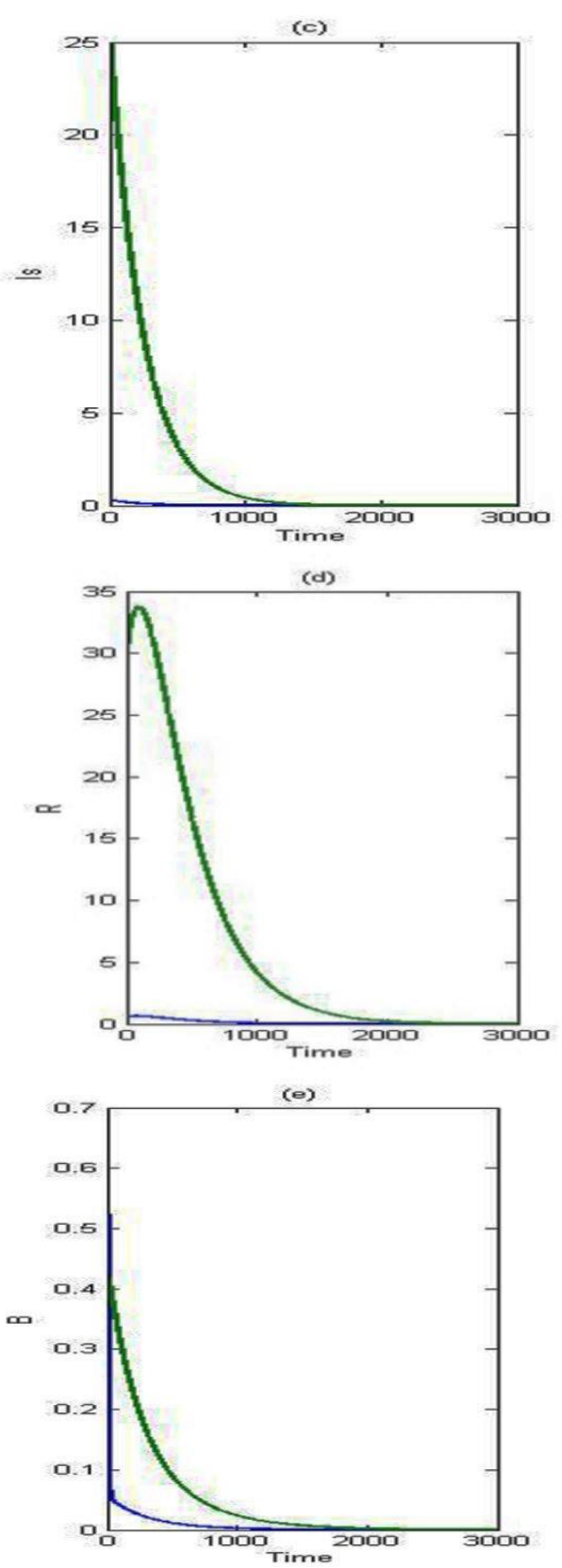

Fig.(1): Time series of the trajectories of system (1) from different initial points for data given in Eq. (13) which show that $E_{0}$ is globally asymptotically stable. (a) For $S$, (b) For $I_{A}$, (c) For $I_{S}$, (d) For $R$, (e) For $B$.

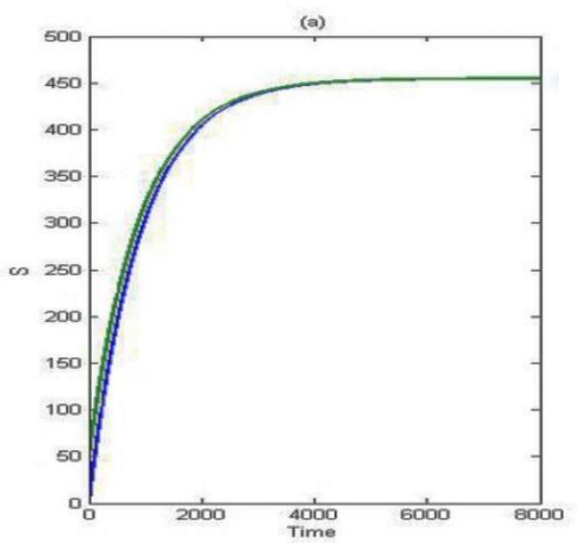

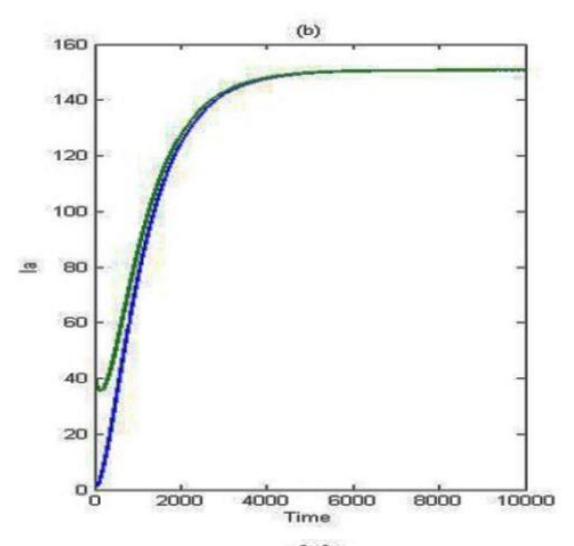
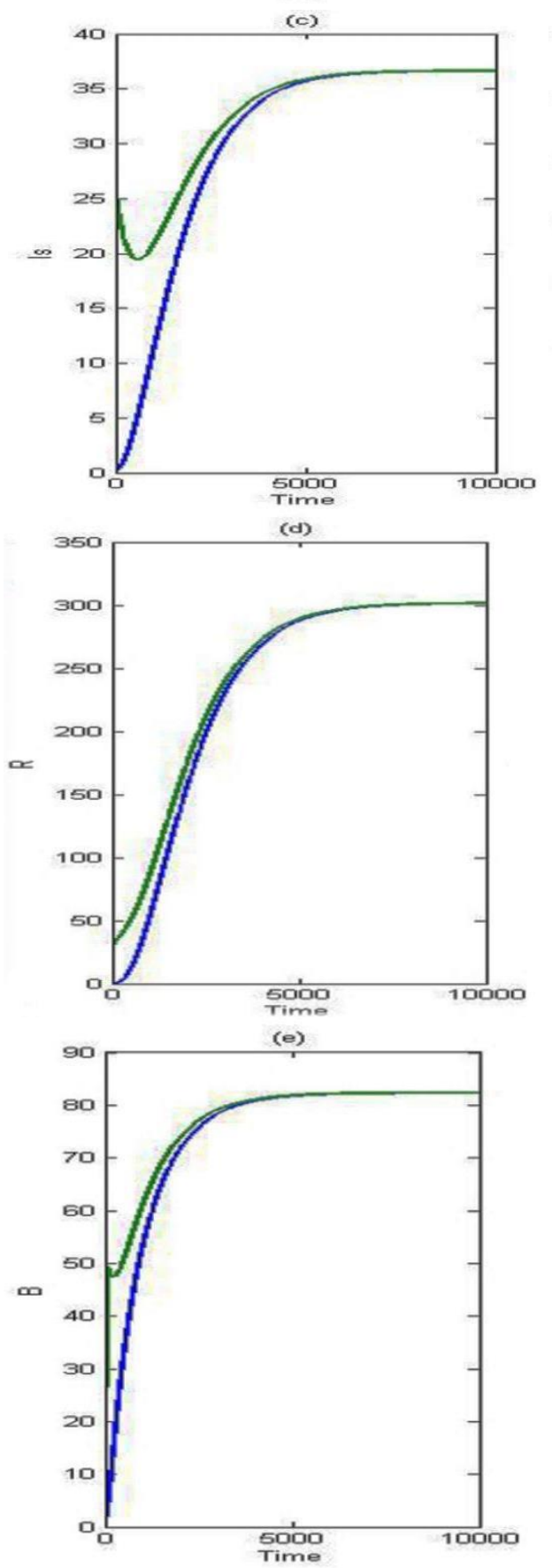

Fig.(2): Time series of the trajectories of system (1) from different initial points for data given in Eq. (14) which show that $E_{1}$ is globally asymptotically stable. (a) For $S$, (b) For $I_{A}$, (c) For $I_{S}$, (d) For R, (e) For $B$. 
Ahmed A. Muhseen
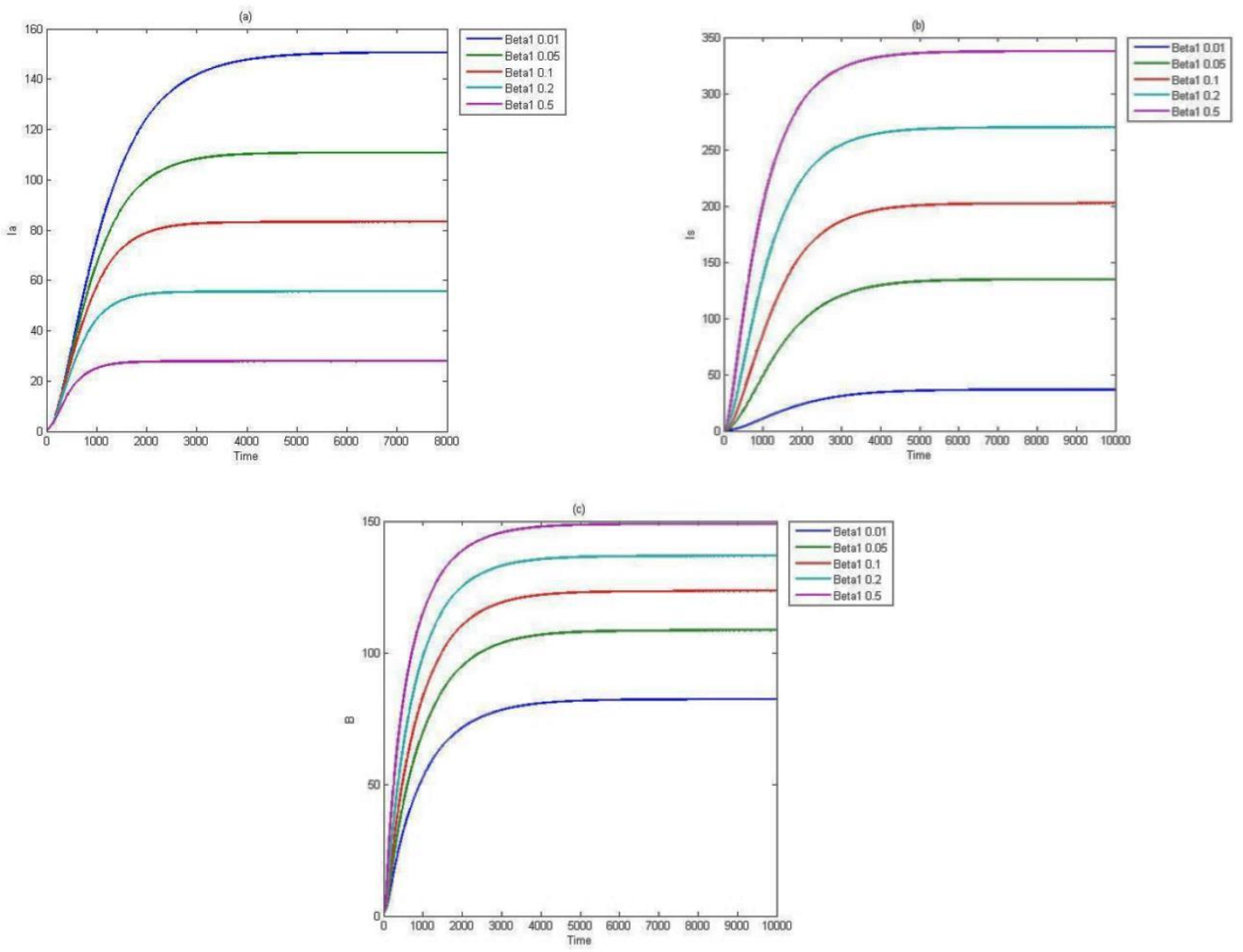

Fig.(3): Time series of the trajectories of system (1). (a) For $I_{A}$, (b) For $I_{S}$, (c) For B.
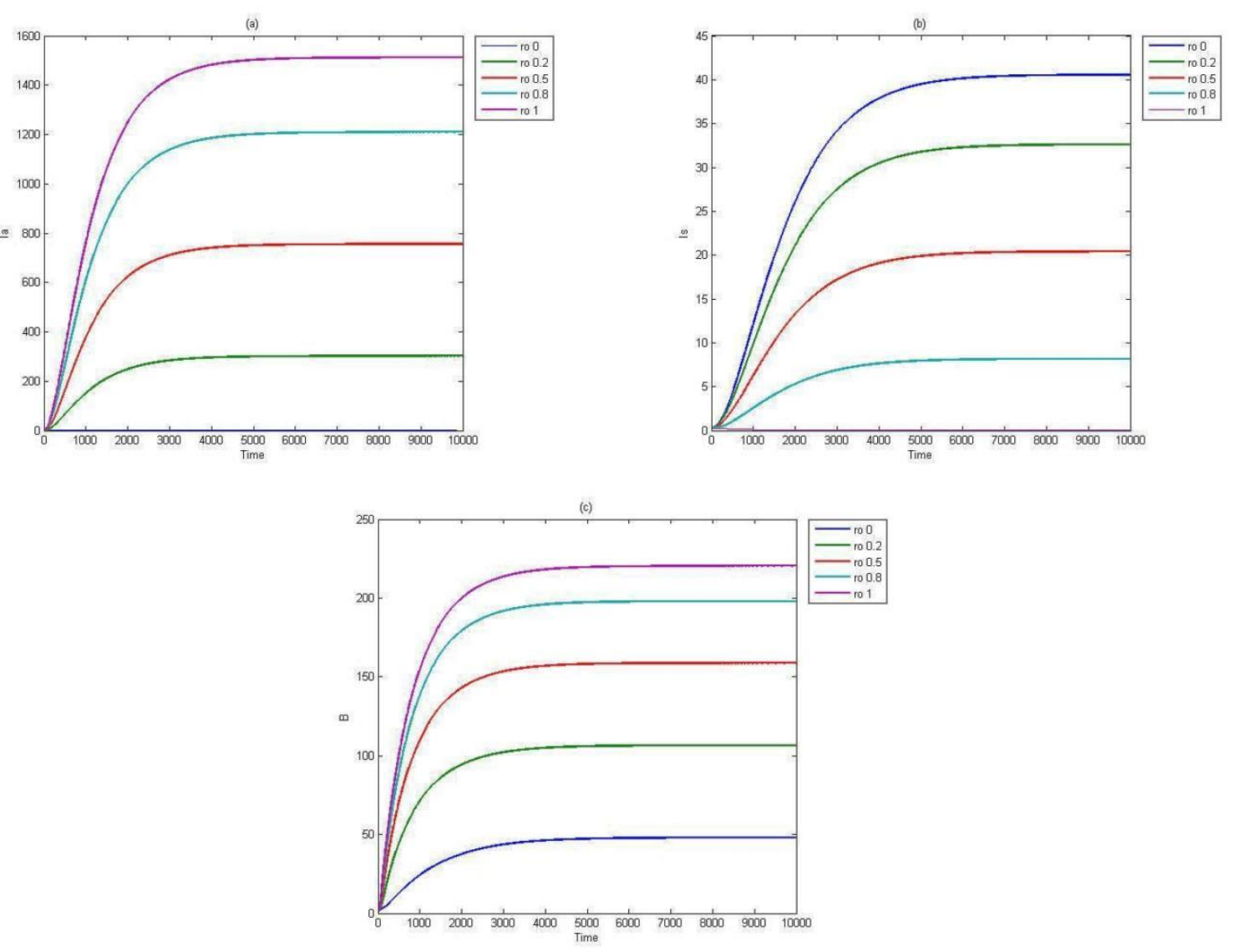

Fig.(4): Time series of the trajectories of system (1). (a) For $I_{A}$, (b) For $I_{S}$, (c) For $B$. 

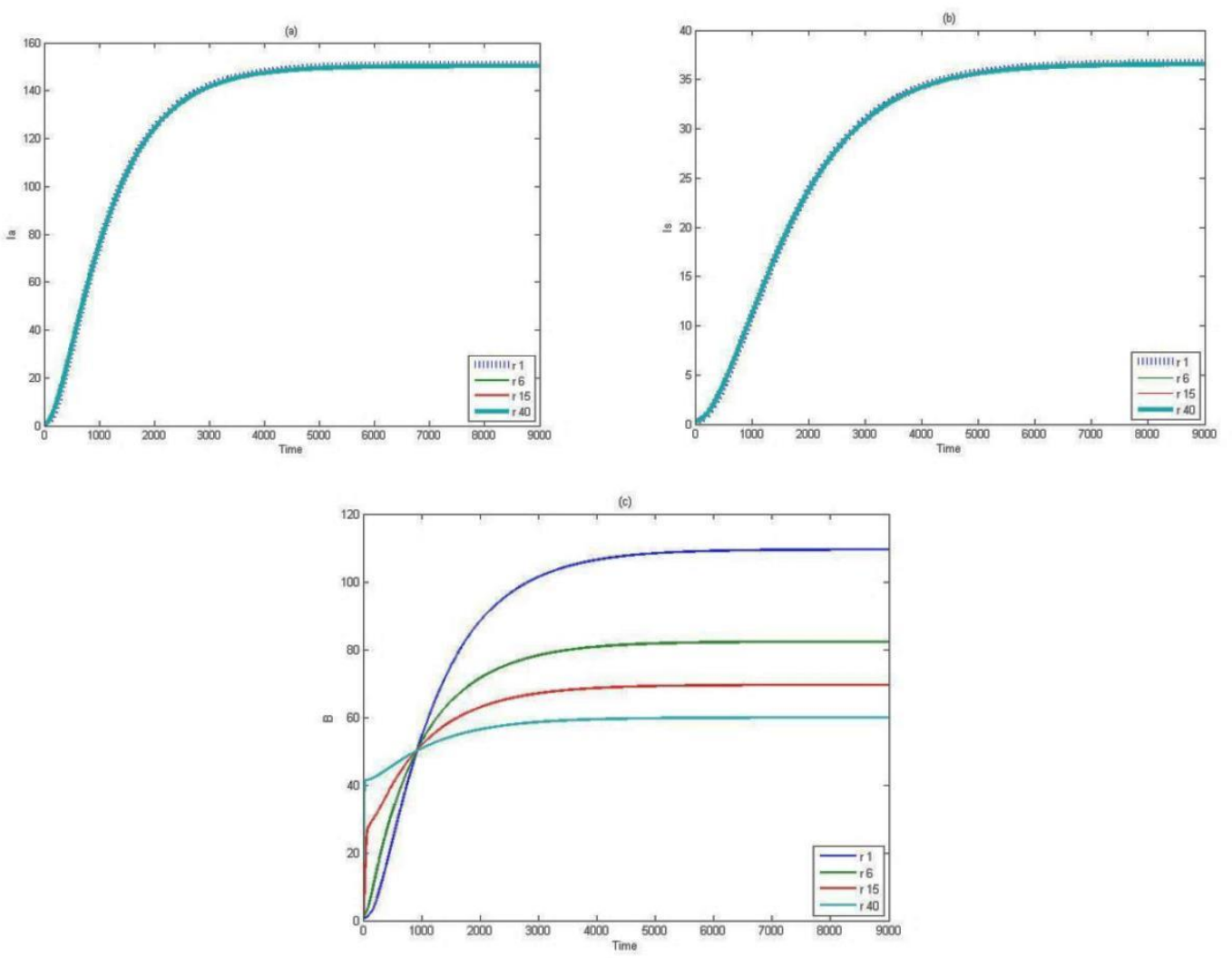

Fig. (5): Time series of the trajectories of system (1). (a) For $I_{A}$, (b) For $I_{S}$, (c) For B.

\section{Conclusion and Discussion}

In this paper, we proposed and analyzed an epidemiological model that described the dynamical behavior of an epidemic model, where the infectious disease transmitted directly from contact between them by Holling type II. The model included five non-linear autonomous differential equations that describe the dynamics of five different populations, namely susceptible individuals $(S)$, asymptomatic infectious individuals $\left(I_{A}\right)$, symptomatic infectious individuals $\left(I_{S}\right)$, removal individuals from infected classes $(R)$ and $B$ is cholera population. The boundedness of system (1) has been discussed. The conditions for existence, stability for each equilibrium points are obtained. Further, it is observed that the disease free equilibrium point $\left(E_{0}\right)$ exists when $I_{A}=I_{S}=B=0$ and it locally stable if the conditions $(9 a-9 b)$ are hold, and then it is globally stable if and only if the conditions (11a-11b) are hold. The endemic equilibrium point $\left(E_{1}\right)$ exists if $\Omega_{4}>0$ and one of three conditions is hold ( $7 \mathrm{a}$ or $7 \mathrm{~b}$ or $7 \mathrm{c}$ ) and locally stable if the conditions (10a-10f) are hold more than it is globally stable if and only if the conditions (12a-12f) hold. Finally, to understand the effect of varying each parameter on the global system (1) and confirm our above analytical results, system (1) has been solved numerically for different sets of initial points and different sets of parameters given by equation (14), and the following observations are made:

1) System (1) do not has periodic dynamic, instead it they approach either to the all equilibrium point.

2) As the incidence rate of disease (contact incidence rate $\left(\beta_{1}\right)$ ) increase, the asymptotic behavior of systems (1) approaching to endemic equilibrium point. In fact are $\left(\beta_{1}\right)$ increase it is observed that the number of $\left(I_{A}\right)$ decrease and the number of $\left(I_{S}\right.$ and $\left.B\right)$ increase.

3) As the fraction rate $(0 \leq \rho \leq 1)$ increase, the asymptotic behavior of systems (1) approaching to endemic equilibrium point. In fact as $(\rho)$ increase it is observed that the number of $\left(I_{S}\right)$ decrease and the number of $\left(I_{A}\right.$ and $\left.B\right)$ increase.

4) As the intrinsic growth rate or carrying capacity of cholera population rate, are increases $\left(r, K_{2}\right)$ respectively the 
asymptotic behavior of systems (1) approaching to endemic equilibrium point with increase it is observed that the numbers of $\left(I_{A}, I_{S}, B\right)$ are increase.

5) As the recovery rate $\left(\gamma_{A}\right.$ or $\left.\gamma_{S}\right)$ increases, then increase it is observed that the numbers of $\left(I_{A}, I_{S}, B\right)$ are decrease.

\section{References}

[1] Alam, LaRocque R.C., Harris J.B., Vanderspurt C., Ryan E.T., Qadri F., and Calderwood S.B., Hyperinfectivity of human-passaged Vibrio cholerae can be modeled by growth in the infant mouse, Infect. Immun. 73, 6674-6679, 2005.

[2] Hendrix T.R., The pathophysiology of cholera, Bull. NY Acad. Med. 47, 11691180, 1971.

[3] Kaper J.B., Morris J.G., and Levine M.M., Cholera, Clin. Microbiol. Rev. 8, 48-86, 1995.

[4] King A.A., Lonides E.L., Pascual M., and Bouma M.J., Inapparent infections and Cholera dynamics, Nature 454, 877-881, 2008.

[5] Merrell D.S., Butler S.M., and Qadri et al. F., Host-induced epidemic spread of the cholera bacterium, Nature 417, 642-645, 2002.

[6] Nelson E.J., Harris J.B., Morris J.G., Calderwood S.B., and Camilli A., Cholera transmission: the host, pathogen and bacteriophage dynamics, Nat. Rev.: Microbiology 7, 693-702, 2009.

[7] Pascual M., Bouma M., and Dobson A., Cholera and climate: revisiting the quantitative evidence, Microbes Infections 4, 237-245, 2002.

[8] Tudor V., and Strati I., Smallpox, Cholera, Abacus Press, Tunbridge Wells, 1977.

[9] Dietz K., The estimation of the basic reproduction number for infections diseases, Stat. Methods Med. Res. 2, 23-41, 1993.

[10] Hethcote H.W., The mathematics of infectious diseases, SIAM Rev. 42, 599653, 2000.

[11] Capasso V., and Paveri-Fontana S.L., A mathematical model for the cholera epidemic in the European Mediterranean

region, Rev. dépidémoligié et de santé Publiqué 27, 121-132, 1979.

[12] Codeco C.T., Endemic and epidemic dynamics of cholera: the role of the aquatic reservoir, BMC Infectious Diseases 1:1, 2001.

[13] Pascual M., Bouma M.J., Dobson A.P., Cholera and climate: revisiting the quantitative evidence, Microbes and Infection 4 (2), 237-245, 2002.

[14] Joh R.I., Wang H., Weiss H., Weitz J.S., Dynamics of indirectly transmitted infectious diseases with immunological threshold, Bulletin of Mathematical Biology 71, 845-862, 2009.

[15] Miller Neilan R.L., Schaefer E., Gaff H., Fister K.R., Lenhart S., Modeling optimal intervention strategies for cholera, Bulletin of Mathematical Biology 72 (8), 20042018, 2010.

[16] Hirsch M. W., and Smale, S., Differential Equation, Dynamical System, and Linear Algebra. Academic Press, Inc., New York. 169-170, 1974.

[17] Van den Driessche P., Watmough J. Reproduction numbers and sub-threshold endemic equilibria for compartmental models of disease transmission. Mathematical Biosciences, 180:29-48. DOI: $\quad 10.1016 / \mathrm{S} 0025-5564(02) 00108-6$ 2002.

[18] Lial M. L., precalculus et. al., Adisonwesly, New York, 2001.

[19] Horn R. A., Johanson C. R., matrix analysis, Cambridge University press, 1985.

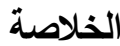

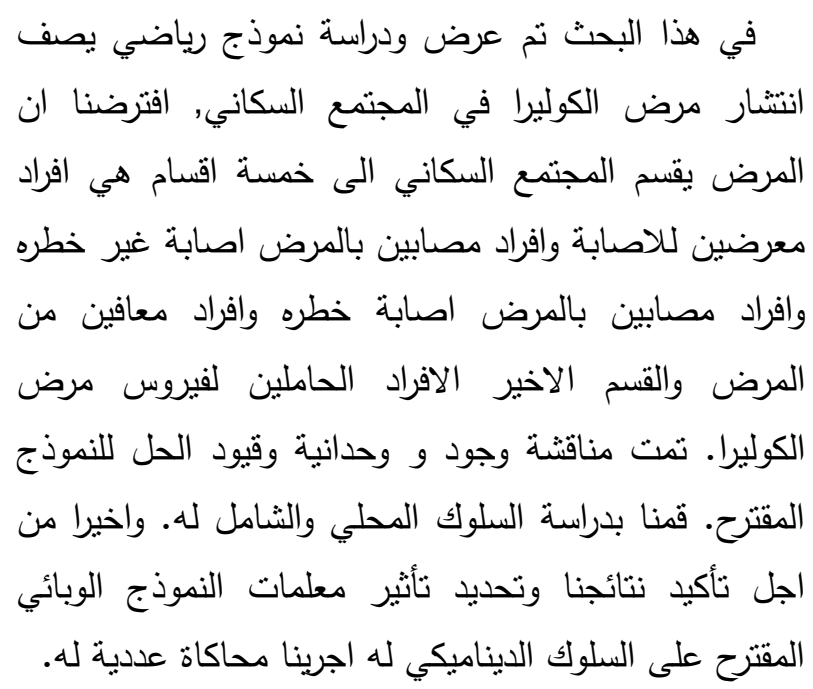

